\title{
SCL-SSC: Supervised Contrastive Learning for Sleep Stage
} Classification

This paper was downloaded from TechRxiv (https://www.techrxiv.org).

\section{LICENSE}

CC BY-SA 4.0

SUBMISSION DATE / POSTED DATE

01-01-2022 / 05-01-2022

CITATION

Kumar, Chandra Bhushan (2022): SCL-SSC: Supervised Contrastive Learning for Sleep Stage Classification. TechRxiv. Preprint. https://doi.org/10.36227/techrxiv.17711369.v1

DOI

10.36227/techrxiv.17711369.v1 


\title{
SCL-SSC: Supervised Contrastive Learning for Sleep Stage Classification
}

\author{
Chandra Bhushan Kumar ${ }^{1}$, Arnab Kumar Mondal ${ }^{2}$, Manvir Bhatia ${ }^{3}$, Bijiya K. Panigrahi ${ }^{4}$, Tapan K. Gandhi ${ }^{4 *}$ \\ ${ }^{1}$ Bharti School of Telecommunication and Management, Indian Institute of Technology, Delhi, New Delhi, \\ India., ${ }^{2}$ School of Information Technology, Indian Institute of Technology, Delhi, New Delhi, India. \\ ${ }^{3}$ Neurology \& Sleep Centre, New Delhi, India. \\ ${ }^{4}$ Department of Electrical Engineering, Indian Institute of Technology, Delhi, New Delhi, India.
}

\begin{abstract}
Sleep data scoring is a crucial and primary step for diagnosis of sleep disorder to know the sleep stages from the PSG signals. Traditionally, sleep data scoring is performed manually through visual inspection by an expert which is a tedious job and requires domain knowledge. So, automatic sleep stage classification is entailed to simplify the process. In this study, we have proposed SCL-SSC(Supervised Contrastive Learning for Sleep Stage Classification), a deep learning-based framework for sleep stage classification which performs the task in two stages, 1) feature representation learning, and 2) classification. The feature learner is trained separately to represent the raw EEG signals in the feature space such that the distance between the embedding of EEG signals of the same sleep stage has less than the distance between the embedding of EEG signals of different sleep stages in the euclidean space. On top of feature learners, we have trained the classifier to perform the classification task. The distribution of sleep stages is not uniform in the PSG data, wake(W) and N2 sleep stages appear more frequently than the other sleep stages, which leads to an imbalance dataset problem. This paper addresses this issue by using weighted softmax cross-entropy loss function and also dataset oversampling technique utilized to produce synthetic data points for minority sleep stages for approximately balancing the number of sleep stages in the training dataset. The performance of our proposed model is evaluated on the publicly available Physionet datasets EDF-Sleep 2013 and 2018 versions. We have trained and evaluated our model on two EEG channels (Fpz-Cz and $\mathrm{Pz}-\mathrm{Oz}$ ) on these datasets separately. The evaluation result shows that the performance of SCL-SSC is the best annotation performance compared to the existing state-ofart deep learning algorithms to our best of knowledge, with an overall accuracy of $94.1071 \%$ with a macro F1 score of 92.6416 and Cohen's Kappa coefficient $(\kappa) 0.9197$. Our ablation studies on SCL-SSC shows that both triplet loss based pre-training of feature learner and oversampling of minority classes are contributing to better performance of the model(SCL-SSC).
\end{abstract}

Index Terms-Sleep Stages, One dimensional convolutional neural network, Recurrent neural network, Longshort term memory cell, Contrastive learning.

\section{INTRODUCTION}

$\mathbf{S}$ LEEP is an important phenomenon to repair and recover our organic systems including our muscles, immune systems, and various other hormones. One person needs to sleep one-third time of their complete life duration. One night's sleep is divided into two stages: rapid eye movement(REM) and non-rapid eye movement(NREM). Usually, there is considerable eye movement during the REM sleep and little or no eye movement during the NREM sleep. The NREM and REM sleep stages repeat in a cycle during complete sleep duration. Usually, NREM sleep appears first in the cycle followed by REM.

REM sleep plays a vital role in memory retention. The transformation of information from short term memory to long term memory is materialised during REM sleep. The NREM sleep, also known as atmospheric sleep, is further divided into four stages, stage 1 to stage 4 according to the R-K scoring manual [1]. It can also be divided into growth hormone production and cell recovery begin stage [2]. The first stage comes between being awake and falling asleep. The second stage is light sleep, when heart rate and breathing regulate and body temperature drops. Stages 3 and 4 are considered as slow-wave sleep (SWS). The survey results had revealed that 5070 million adults having 70 different sleep disorders and reports of 60 percents adults having a sleep disorder a few nights a week or more in the USA [3], [4]. Sleep disorders affects the health and as well as quality of life. The short-term bad effects are the impaired attention and concentration, reduction in quality of life continuously, reduction in productivity due to increase in absenteeism rates, and also it increases the chance of accident at work, home, or while driving and the long term bad affects are coronary artery disease, heart failure, high blood pressure, obesity, type 2 diabetes mellitus, stroke, memory impairment, and depression and also it increases the morbidity and mortality due to increase in automobile accidents [5]. Therefore it is crucial to diagnose the sleep disorders in early stages.

Sleep scoring is the first step in analysing sleep quality assessment and diagnosis. The PSG signals are cut into a fixed length of 30second which are defined as epochs [6], [7], [8]. In medical practice, sleep scoring is done manually by a sleep expert according to the standard guidelines are suggested in the American Academy of Sleep Medicine (AASM) [7] or the Rechtschaffen and Kales standard [9]. Sleep experts need to analyse approximately 8 hours PSG signals for sleep scoring, which is a cumbersome process.

The research community tried to address this problem by proposing automatic sleep scoring techniques. These proposed techniques can be grouped into two categories; traditional machine learning methods based algorithms and advanced deep learning based algorithms. The traditional machine learning approaches have been proposed for the sleep stage classification using hand-crafted features. Usually, they first preprocess EEG signals to remove artifacts and noise; then extract and select discriminative features. Finally, machine learning models are trained for the classification task. In [10], [11], and [12] authors have extracted conventional time, frequency, and timefrequency domain handcrafted features from EEG signals. Then, the traditional machine learning algorithm like support vector machine (SVM) [13], random forest [14], and neural network [15] are used for classification. The performance of these algorithms is highly dependent on the quality of hand-crafted features that need prior domain expertise, limiting the scope to optimize the performance of these approaches.

In contrast to these traditional approaches, deep learning provides an end-to-end solution that automates the process of finding a relationship between the raw input signal and the output. The application of deep learning in area such as image processing [16], video analysis [17], natural language processing [18], [19], and so on has 
attened great success. Recently, many deep learning approaches like autoencoders [20], convolutional neural networks (CNNs) [21], and recurrent neural networks (RNNs) [22], have been employed for sleep stage classification and has performed better than the conventional machine learning approaches.

In [23], a generative model is combined with a discriminative model for sleep stage classification using EEG, EMG, and EOG, where 28 handcrafted features are used as an input to the deep belief network (DBN). In [24], the predictive performance of traditional logistic regression is compared with the recent deployed advanced deep learning algorithms such as multilayer perceptron (MLP), convolutional neural network $(\mathrm{CNN})$, simple Elman-type recurrent neural network (RNN), long short-term memory (LSTM-RNN), and a time-batched version of LSTM-RNN (TB-LSTM) on actigraphy data and had reported that $\mathrm{CNN}$ had the highest performance. In [25], attention mechanism based recurrent neural network, a novel compact convolution neural network(C-CNN), and bidirectional long-short term memory(BiLSTM) were proposed for automatic sleep staging using single-channel EEG signal. In the recurrent neural network utilized an attention mechanism to learn the most significant part in the signals and bidirectional long-short term memory(BiLSTM) uses 1D CNN for feature extraction from single channel EEG signals and established that this network provides better performance than $\mathrm{C}$ $\mathrm{CNN}$ in the cost of longer training time. In [23], a mixed neural network was proposed for sleep stage classification where hierarchical features from the EEG and the EOG signals were captured using rectifier neural network and long short-term memory network was used as a classifier.The population heterogeneity present in the EEG signals during sleep due to influence of different cranial structures and demographic location and also the sleep stage classification is sequential problem where thr past epochs influences the present epochs [7], [26]. A mixed neural network was proposed in [26] to adrees the problem of population heterogeneity in data and for recognition of temporal patterns present in the data. The proposed model is composed of dense layer of rectifier neural network and longshort term memory(LSTM). In [27], long-short-term memory(LSTM) based network were used to capture temporal information for classification of sleep tates(sleep/wake) and detecting sleep onset/offset using the multimodal signals such as physiological and behavioral data.The DeepSleepNet was proposed in [22], where time-variant features were extracted using CNN-based network and bidirectional long-short term memory(LSTM) cell were used for sleep stages classification automatically from EEG signals. SeqSleepNet, a hierarchical recurrent neural network, was proposed in [28], where filter bank layer was used to learn frequency-domain filter and an attentionbased recurrent layer was used for designing short-term sequential modeling. In [29], an automatic sleep stage classification method known as SleepEEGNet was proposed that uses single-channel EEG signals. In this method, a deep convolution neural network (CNN) was used to capture time-invariant features and frequency information from EEG signal; an attention network was used to learn the most significant part in the input and bidirectional recurrent neural network for learning dependencies between the epochs of EEG signals for scoring. A 34-layer deep residual convolutional architecture was proposed in [30] for end-to-end sleep staging using raw EEG signals. Recently, a novel attention-based deep learning architecture called AttnSleep, was proposed for automatic sleep stage classification in [31]. The architecture of AttnSleep can be seperated in the two modules: first, $\mathrm{CNN}$ based feature extractor, and second, the temporal context encoder(TCE). The feature extractor composed of a multiresolution $\mathrm{CNN}$ module and an adaptive feature recalibration tyo learn interdependencies, and the TCE module utilizes the concept of multi-head self-attention mechanism with causal convolutions to learn the temporal interdependencies.

As discussed above, sleep stage classification is a five-class classification problem with classes: wake, $N_{1}, N_{2}, N_{3}$, and REM sleep. These sleep stages appear in a cycle. We believe that PSG signals are sequential data. So, the learning correlation between the sleep stages will enhance the classification performance of the model. In this paper, we propose a model that will learn the correlation between different sleep stages. The proposed model consists of the following building blocks:

1) Feature learner block: This block is separately trained to learn the similarity between the same classes of sleep stages and dissimilarity between different classes of sleep stages.

2) Bidirectional recurrent neural network(BiRNN) based encoderdecoder model for capturing temporal information from past, present, and future sequences.

3) Self-attention mechanism is applied to learn the most relevant part from the output of the decoder sequence.

The main contributions of our work are the following.

- We have deployed CNN based feature learner model to learn the features from the single-channel EEG signals.

- In the first phase of training, we train the feature learner model to learn the similarity between the same class of sleep stages and dissimilarity between the different class of sleep stages with the help of the triplet loss function.

- In the second phase of training, we deploy a sequence-tosequence bidirectional recurrent neural network(BiRNN) with an attention mechanism for scoring sleep data with better accuracy.

- We have also analysed the relevance of using the triplet loss function for the sleep stage classification task using singlechannel EEG signals.

- The proposed model is an end-to-end deep learning model for sleep stage classification using raw single-channel EEG signals as an input without using any hand-crafted features.

The remaining portions of this paper will discuss the methodology and experimental results followed by result discussion.

\section{Methodology}

This section provides a detailed description of our proposed deep learning model developed for automatic sleep stage classification.

\section{A. Data Pre-processing}

The raw PSG data contains many types of physiological signals for example EEG, EOG, EMG, etc. We utilize single-channel EEG signals, which is segmented into epochs of length 30 -seconds. We have performed the following simple two steps to achieve that:

1) The continuous raw single-channel EEG signals segmented in epochs of 30-seconds and labels were assigned to each epoch based on annotation files.

2) Each 30-seconds epoch normalised to zero mean and unit standard deviation which increase the convergence rate of learning model.

We have performed these two simple pre-processing steps without any form of filtering and noise removal.

\section{B. Model Architecture}

In this section, we will describe our proposed model architecture in detail. The proposed methodology is presented in figure 1. We propose a two stage training process. In the first phase we train a deep neural network called feature learner $f_{\theta}$, which is responsible for rich representation learning and extracts expressive features from the raw EEG signals. In the second stage we utilise the pretrained feature learner from the first stage for feature extraction and train a classifier that is responsible for classification of sleep stages. 


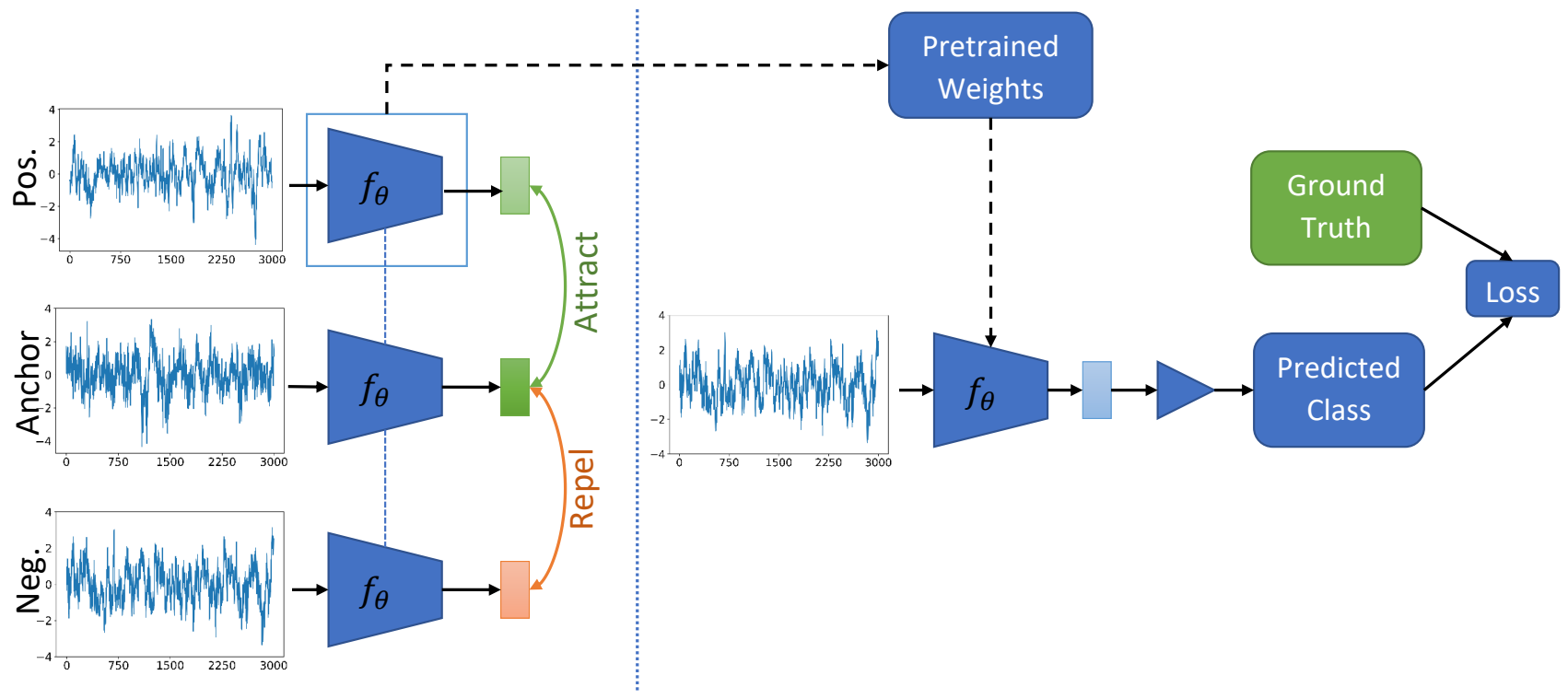

Fig. 1: Illustration of our proposed method.

1) Feature Representation Learning Stage: The purpose of this part of the model architecture is to train a feature learner function $f_{\theta}(x)$ to learn feature representation for transforming the raw EEG signals to a compact Euclidean space where distance is the measurement metric for similarity and dissimilarity.

There is a trade-off between time domain and frequency domain features as mentioned in [32]. To overcome the time domain and frequency domain trade-off, we have utilized same 1D CNN architecture for feature learning as in [22], [29].The architecture of feature learner uses two arm structure: one with small size filters and other with large size filters of $1 \mathrm{D} \mathrm{CNN}$ in parallel where small size filters are being used to extract time domain features and large size filters are being used to extract frequency domain features. The architecture consists of four 1D CNN layers with Rectified linear unit (ReLU) as an activation function in both side of parallel part. In each part, first layer is $1 \mathrm{D} \mathrm{CNN}$ that is followed by max pooling layer and dropout layer, and then three consecutive 1D CNN layers followed by max pooling layer. In the next, outputs of both parallel part have concatenated and then dropout layer have been used before output of feature learner. The input of this sub-model is 30-seconds epoch of raw EEG signals.

In our experimental setup, we have trained feature learner $f_{\theta}(x)$ to transform the EEG signal to a embedding space using three sets of data: reference data, positive data, and negative data, as shown in figure 1. These three sets of data is sampled from single data set, each set contains same number of examples. The same index of reference data set and positive data sets contains raw EEG signals with same sleep stage label, however different examples and negative data sets contains signals with different sleep stage label at the corresponding index. Now, with this setup the feature learner model is trained separately to learn the embedding using the triplet loss function. The concept of triplet loss function introduced in [33] for the first time. The magical power of triplet loss function has been known to the deep learning research community after it was used in FaceNet [34] where triplet loss function was used to train the model to learn embedding from face images in face recognition task.

2) Triplet Loss Function and its Intuition: The feature learner $f_{\theta}(x)$ transforms the EEG signal $x$ to a feature vector in $\mathbb{R}^{d}$. In the feature space, we would like to have Euclidean distance $d\left(R_{a}, R_{p}\right)$ between anchor data representation $\left(R_{a}\right)$ and positive data representation $\left(R_{p}\right)$ always less than the Euclidean distance $d\left(R_{a}, R_{n}\right)$ between anchor data representation $\left(R_{a}\right)$ and negative data representation $\left(R_{n}\right)$ by a margin of $\alpha$. So, we can write,

$$
\left(d\left(R_{a}, R_{p}\right)+\alpha\right)<d\left(R_{a}, R_{n}\right)
$$

where $d\left(R_{a}, R_{p}\right)$ and $d\left(R_{a}, R_{n}\right)$ are defined as:

$$
\begin{aligned}
& d\left(R_{a}, R_{p}\right)=\sqrt{\left(\left\|R_{a}-R_{p}\right\|\right)^{2}} \\
& d\left(R_{a}, R_{n}\right)=\sqrt{\left(\left\|R_{a}-R_{n}\right\|\right)^{2}}
\end{aligned}
$$

Now, inspired from this simple condition given in equation 1 , the triplet loss is defined as follows:

$$
L\left(R_{a}, R_{p}, R_{n}\right)=\max \left(0, \alpha+d\left(R_{a}, R_{p}\right)-d\left(R_{a}, R_{n}\right)\right)
$$

After analyzing the equation (4), we can reach to the following conclusion:

- if $\left(d\left(R_{a}, R_{p}\right)+\alpha\right)<d\left(R_{a}, R_{n}\right)$, then loss will be zero.

- if $d\left(R_{a}, R_{p}\right)<d\left(R_{a}, R_{n}\right)<d\left(R_{a}, R_{p}\right)+\alpha$, then positive data is more closer to anchor data than negative data in Euclidean space but the margin between distances is less than $\alpha$. Therefore loss will have positive value but less than $\alpha$.

- if $d\left(R_{a}, R_{p}\right)=d\left(R_{a}, R_{n}\right)$, then loss will be equal to $\alpha$.

- if $d\left(R_{a}, R_{p}\right)>d\left(R_{a}, R_{n}\right)$, then loss will have positive value more than $\alpha$.

- Here, the $\alpha$ is acting as bias that helps feature learner minimize the distance between EEG signals of same sleep stages (distance between reference data and positive data) in the feature space whereas maximizing the distance between EEG signals of different sleep stages (distance between reference data and negative data) in the feature space. 
Now, we can say that the triplet loss based learning forces the feature learner to embed the EEG signals of same sleep stages within a close neighbourhood in the feature space. Whereas the distance between EEG signals of different sleep stages in the feature space gets maximized.

3) Classifier: In this part of the model architecture, we have used feature learner $f_{\theta}(x)$ that is trained in the feature representation learning part of the model and the attention mechanism-based sequence to sequence classifier for categorizing the sleep stages as shown in the figure (1). Here, we have used a trained feature learner to represent EEG signals epoch of 30 -seconds in embedding space. Now, the embedding of raw EEG signal is fed to the encoder-decoder-based classifier with self-attention mechanism for final classification.

The basic sequence to sequence model is encoder-decoder based model, where encoder encodes source input signals into a fixed length encoded vector. The fixed length encoded vector is nothing but the hidden states of the encoder at last time step that passed as an input to the decoder at each time step. Then the categorized outputs are produced by the decoder from fixed length encoded vector. Although, the hidden states of encoder are updated at each time steps of encoder and the last update is done at the last time step of the encoder.

We have proposed an encoder-decoder-based classifier with selfattention, where embedding of EEG signals are fed to the encoder and where encoder encodes source inputs into a fixed length encoded vector. The fixed length encoded vector is nothing but the hidden states of the encoder at last time step that passed as an input to the decoder at each time step. Then the hidden states of each time state is passed to the attention block where self-attention mechanism is utilized to learn the dependencies between the hidden states of each time step of the decoder and is focused on more relevant part of the hidden states. At last, the output of attention block is fed to the softmax layer to produce the probability distribution of sleep stages at each time step that corresponds to the class of each epoch of 30seconds EEG signals.

The building blocks of the encoder and decoder are the bidirectional long-short term memory(LSTM) cell, which is capable of learning long-term context-dependency within the time series data. Since the PSG data is sequential data so there must be some correlation between the different epochs of EEG signals, and by learning correlation between different epochs the performance will improve. So, to learn the correlation between different sleep stages, an encoderdecoder-based classifier with self-attention is a perfect choice for classification tasks where the encoder is capable of learning the longterm context dependency present in the input and also in capturing non-linear dependency in the entire series. The bi-directional longshort term memory(Bi-LSTM) shares the information of both past and future time steps with current time steps. In the Bi-LSTM based encoder, the hidden states of both past and future time steps are passed to the current time step for sharing the information from the both previous and future sequences that avoids the limitation of LSTM based encoder that shares the previous sequence information with the current sequence only. The Bi-LSTM based encoder is composed of two networks forward and backward network. The forward network processes information in forward direction using input in normal time sequence, $\mathrm{t}=1,2, \ldots ., \mathrm{T}$, and the backward network processes the information in backward direction using input in reverse order, $\mathrm{t}=\mathrm{T}, \mathrm{T}-1, \ldots . ., 2,1$. The output of encoder is the weighted sum of outputs of forward and backward networks, that can be written as below:

$$
\overrightarrow{h_{t}}=\tanh \left(\vec{W} x_{t}+\vec{V} \overrightarrow{h_{t-1}}+\vec{b}\right)
$$

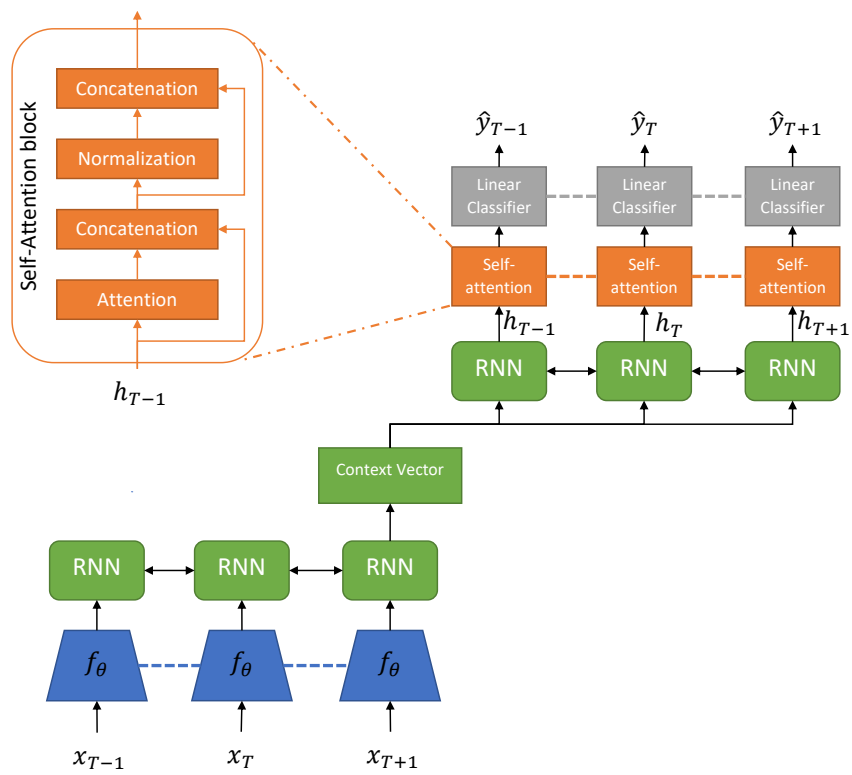

Fig. 2: Illustration of an encoder-decoder-based classifier with selfattention. The weight sharing between blocks shown by dotted line between them.

$$
\begin{gathered}
\overleftarrow{h_{t}}=\tanh \left(\overleftarrow{W} x_{t}+\overleftarrow{V} \overleftarrow{h_{t+1}}+\overleftarrow{b}\right) \\
y_{t}=\left(U\left[\overrightarrow{h_{t}} ; \overleftarrow{h_{t}}\right]+b_{y}\right)
\end{gathered}
$$

where $\left(\overrightarrow{h_{t}}, \vec{b}\right)$ and $\left(\overleftarrow{h_{t}}, \overleftarrow{b}\right)$ are the hidden state and bias of forward network and backward network at time step t respectively.

In the attention block, we have utilized self-attention mechanism to learn the dependencies between the input sequence that is the hidden states of each time step of the decoder. The operations of self-attention mechanism can be formulated as:

$$
\alpha=\operatorname{softmax}\left(W_{s 2} \tanh \left(W_{s 1} H^{T}\right)\right)
$$

where $W_{s 2}$ and $W_{s 1}$ are the weight vectors, and $\mathrm{H}=\left(h_{1}, h_{2}, \ldots, h_{n}\right)$ is the input vector and $\alpha=\alpha_{1}, \alpha_{2}, \ldots, \alpha_{n}$ is the calculated attention score.

$$
\operatorname{attention}(H)=\alpha H^{T}
$$

from equation (8), we can say that the self-attention is multiplicative kind of attention mechanism. So, we have utilized the Loung attention mechanism [35] for designing self-attention mechanism where the hidden state vector $\mathrm{H}$ is fed two times as query and key.

Here, in Loung attention mechanism, the score between the hidden state $h_{i}$ of the decoder at time step $i$ and the hidden state $h_{t-1}$ of the decoder at time step $t-1$ is calculated first. The score is nothing but the content-based function $f\left(h_{t-1}, h_{i}\right)$ that is defined below:

$$
f\left(h_{t-1}, h_{i}\right)=\tanh \left(W_{h} h_{t-1}+W_{a} h_{i}\right)
$$

where $W_{h}$ and $W_{a}$ are the weight vector.

The importance of hidden state is defined in the terms of attention weight which is in the range[0,1], the lowest value 0 is assigned to the unrelated hidden state and the highest value 1 is assigned to the most relevant hidden state of the encoder, for producing target at current time step of the decoder. So, the attention weight $\alpha_{i}$ can be treated as the probability corresponding to the importance of hidden state at time step $i$ of the encoder which is defined as: 


$$
\alpha_{i}=\operatorname{softmax}\left(f\left(h_{t-1}, h_{i}\right)\right)=\frac{\exp \left(f\left(h_{t-1}, h_{i}\right)\right)}{\sum_{j=1}^{n} \exp \left(f\left(h_{t-1}, h_{j}\right)\right)}
$$

where i $\epsilon 1,2, \ldots, n$ Finally, the context vector $c_{t}$ at time step $t$ in the decoder is defined as the weighted sum of all the hidden states of the encoder. The context vector $c_{t}$ is formulated as:

$$
c_{t}=\sum_{i=1}^{n} \alpha_{i} h_{i}
$$

In the attention mechanism compares the previous target hidden state $h_{t-1}$ with each hidden states of the decoder by computing score value $f\left(h_{t-1}, h_{i}\right)$ as in equation(10), then the attention weight $\alpha_{i}$ as in equation(11) is calculated for time step $i$ of the decoder and is repeated for $n$-time steps of the decoder. In the last, context vector $c_{t}$ is calculated for time step $t$ in the decoder as in equation(12) and repeated for all time steps in the decoder for producing the targets.

The output of self-attention layer is concatenated with the input vector as shown in the figure 2. Finally, the concatenated vector is fed to the normalization layer and again the normalized output is concatenated with the input of the normalization layer as shown in the block diagram in figure 2 .

4) Loss Function: As we know that some of the sleep stages are more frequent than others, which leads to an imbalanced sleep data set like other biomedical data sets. In these kind of cases, the contribution of majority classes to the normal loss functions like mean square error(MSE) are more than minority classes while training the model, which forces the model to be biased towards the majority classes. To overcome this problem, a special kind of loss function called mean false error(MFE) and mean square false error(MSFE) were introduced in [36] for training deep neural network on imbalanced class data to cancel out the dominating effect of the majority classes. The extension of MFE and MSFE loss functions for multi-class classification task were used for sleep stage classification in [29]. We have utilised the weighted softmax cross-entropy loss that is given in the following equations:

$$
\begin{gathered}
l\left(c_{i}\right)=-\frac{1}{C_{i}} \sum_{j=1}^{C_{i}} y_{c_{i}}^{j} \log \hat{y}_{c_{i}}^{j} \\
l_{M F E}=\sum_{i=1}^{N} l\left(c_{i}\right) \\
l_{M S F E}=\sum_{i=1}^{N}\left(l\left(c_{i}\right)\right)^{2}
\end{gathered}
$$

where $c_{i}$ represents the class(i. e. sleep class like w, N1, ..etc.), the $l\left(c_{i}\right)$ denotes the error of the class $c_{i}, C_{i}$ is the total number of example that belongs to the class $c_{i}$, and $\mathrm{N}$ is the number of available classes. We have utilized same loss function as in [29] where the contribution of majority and minority classes are the same.

\section{Datasets and Data Preparation}

We have used the Physionet sleep-edf dataset ( [37], [38]) for the training and evaluation of our proposed model. This dataset is available in two versions: version 1 that was made available in 2013 and version 2 that was introduced in 2018. We will call version 1 and 2 as Sleep-edf-2013 and Sleep-edf-2018 respectively for the sake of simplicity, same as in [29]. The Sleep-edf-2013 contains PSG signals recording of 61 persons and corresponding annotated file and the Sleep-edf-2018 contains PSG signals recording of 197 persons. The epochs of 30-seconds signals were manually scored by well-trained technicians according to the R-K scoring manual [1] of both the datasts. Each records of these datasets contains EEG(Fpz-Cz and Pz-Oz electrode signals), horizontal EOG, chin EMG signals, and event marker. The *PSG.edf files contains the EEG(Fpz-Cz and Pz$\mathrm{Oz}$ electrode signals), horizontal EOG, and chin EMG signals and *Hypnogram.edf files contains the annotations of the sleep pattern. The annotations consists of wake(W), rapid eye movement(R), N1, $\mathrm{N} 2, \mathrm{~N} 3, \mathrm{~N} 4, \mathrm{M}$ (movement time), and ?(not scored). Both datasets consists of the data from two different studies: 1) study of effect of age on the sleep in sleep cassette(CS) study where 20 hours recording of PSG signals is performed on healthy caucasians age group 25-101; 2) study of the temazepam effects on sleep where PSG signals of about 9 hours from 22 caucassian males and femals were recorded for two nights.

We have merged N4 sleep stage to the N3 sleep stage and removed $\mathrm{M}$ and ? part from the data according to the guidelines given in the AASM [7].

\section{EXPERIMENTAL RESULTS}

\section{A. Experimental design}

The frequency of each class is not uniform in the PSG dataset. For example the $\mathrm{W}$ and $\mathrm{N} 2$ class appears more frequently than other classes. So, the PSG data is unbalanced in nature where some sleep stages are much greater in the number than other sleep stages. In case of imbalance dataset, the traditional machine learning algorithms are generally biased towards the majority class and do not perform well. We have addressed this problem by using the weighted softmax cross entropy loss as explained in the loss function section. In addition, we have oversampled dataset using minority over-sampling technique (SMOTE) to produce synthetic data points for minority sleep stages with the help of similarity between existing minority data points [39].

Our proposed model is trained in k-fold cross-validation setup. In the $\mathrm{k}$-fold cross-validation setup, the whole data is divided in $\mathrm{k}$ subset known as $\mathrm{k}$ folds and then the training and testing of the model is performed k-folds. In each unique fold of the training and testing of the model, unique fold of data is used as test data and remaining $\mathrm{k}-1$ folds of data used as training data and this process is repeated k-times. In the last, all the evaluation results are combined.

The RMSProp optimization function have been utilized to minimize mean false error loss $\left(l_{M} F E\right)$ with mini-batch size of 20 and learning rate, $\alpha=0.001$. The weights of the model were $L_{2}$ regularized with $\beta=0.001$ to avoid overfitting of the model.

\section{B. Evaluation Metrics}

The performance of proposed model were evaluted using the metrices like sensitivity(Recall), Specificity, Precision, overall accuracy, and $F_{1 \text { score }}$ which are:

$$
\begin{gathered}
\text { Sensitivity }=\frac{T P}{T P+F N} \\
\text { Specificity }=\frac{T N}{T N+F P} \\
\text { Precision }=\frac{T P}{T P+F P} \\
\text { Accuracy }=\frac{T P+T N}{T P+T N+F P+F N} \\
F_{1 \text { score }}=2 \times \frac{\text { Sensitivity } \times \text { Specificity }}{\text { Sensitivity }+ \text { Specificity }}
\end{gathered}
$$

where TP (True Positive):number of predicted sleep stages as true sleep stages, TN (True Negative): number of predicted sleep stages truly identified as not belonging to the sleep stages, FT(False True): 
TABLE I: Class-wise evaluation metrics

\begin{tabular}{lrrrr}
\hline & precision & recall & f1-score & support \\
\hline $\mathrm{W}$ & 0.986264 & 0.964307 & 0.975162 & 8265 \\
$\mathrm{~N} 1$ & 0.790093 & 0.910128 & 0.845873 & 2804 \\
$\mathrm{~N} 2$ & 0.963792 & 0.931682 & 0.947465 & 17799 \\
$\mathrm{~N} 3$ & 0.877117 & 0.962476 & 0.917816 & 5703 \\
$\mathrm{REM}$ & 0.958605 & 0.933264 & 0.945765 & 7717 \\
macro avg & 0.915174 & 0.940371 & 0.926416 & 42288 \\
weighted avg & 0.944031 & 0.941071 & 0.941833 & 42288 \\
accuracy & & & 0.941071 & 42288 \\
\hline
\end{tabular}

number of stages falsely predicted as sleep stages, FN(False negative): number of predicted sleep stages falsely identified as not belonging to the sleep stages. Apart from this we have also computed Cohen's Kappa coefficient $(\kappa)$ that measures agreement between two algorithms. In this study, the predicted sleep stages are treated as one rater and target sleep stages are treated as another rater for computation of Cohen's Kappa coefficient $(\kappa)$.

\section{Results and Discussion}

1) Effect of Training feature Learner Using Triplet Loss: As mentioned in above section, we have trained feature learner to learn the representation of raw EEG signals in embedding space such that the distance between the embedding of EEG signals with same sleep stages are less than the embedding of EEG signals with different sleep stages in Euclidean space. We have visualized the raw EEG signals and their representation in embedding space using t-SNE plot [40] as shown in figure (3). In figure (3)(a), the t-SNE plot of epochs of raw EEG signals is shown where its clearly visible that the data points with different sleep stage labels are mixed together which indicates that the distance between the epochs with same sleep stage labels can't be differentiated from the distance between the epochs with different sleep stage labels. In figure (3)(b), the t-SNE plot of embedding vectors of raw EEG signals is shown where the data point of each sleep stage label can be visualized easily because almost all the data points of the embedding belongs to the same sleep stage labels are closer than the data points of the embedding belongs to the different sleep stage labels that is the data points belongs to same class are clustered together. Now, we can say that our feature learner is able to represent the raw EEG signals belonging to same sleep stage more closer compared to the signals belonging to different sleep stage in Euclidean space that solve our purpose of training feature learner using triplet loss before retraining with classifier.

2) Classification Performance of Proposed Model: Table I and II are the class-wise performance and the overall accuracy of proposed model using Fpz-Cz EEG channel and Pz-Oz EEG channel of the EDF-Sleep 2013 database respectively. The proposed model achieved $94.1071 \%$ overall accuracy. Here, we can see that the precision, recall, and f1-score values is less for N1 sleep stage and other sleep stages. It may be because the N1 sleep stage is in minority in the database. The confusion matrix produced by the proposed model using Fpz-Cz EEG channel and Pz-Oz EEG channel of the EDF-Sleep 2013 database is shown in figure 4(a) and 4(b) respectively where the diagonal elements are the true positive(TP) value and the values are the class-wise accuracy achieved by the proposed model. As we can see the accuracy of Wake(W), N1, N2, N3, and REM are 96.43\%,91.01\%,93.17\%,96.25\%, and $93.33 \%$ respectively which implies that accuracy is not much affected on the basis of whether the class belongs to majority or minority.

In biomedical applications, there are two methods for evaluating any deep learning model: 1) Intra-patient paradigm, and 2)Interpatient paradigm. In intra-patient paradigm, model is trained and tested on data from the same patient. Training and testing of model is
TABLE II: Class-wise evaluation metrics for Pz-Oz EEG channel

\begin{tabular}{lrrrr}
\hline & precision & recall & f1-score & support \\
\hline W & 0.97 & 0.97 & 0.97 & 8265 \\
N1 & 0.81 & 0.87 & 0.84 & 2804 \\
N2 & 0.96 & 0.93 & 0.95 & 17799 \\
N3 & 0.89 & 0.94 & 0.91 & 5703 \\
REM & 0.94 & 0.95 & 0.95 & 7717 \\
macro avg & 0.92 & 0.93 & 0.92 & 42288 \\
weighted avg & 0.94 & 0.94 & 0.94 & 42288 \\
accuracy & & & 0.94 & 42288 \\
\hline
\end{tabular}

performed on data from different patients in inter-patient paradigm. The inter-patient paradigm is more realistic. So, we have used interpatient paradigm for evaluating our proposed model.

In figure (5), the performance graphs of proposed model on $\mathrm{Fpz}-\mathrm{Cz}$ EEG channel of EDF-Sleep-2013 database are shown for randomly selected folds of the training.

The performance of our proposed model is compared with the other existing state-of-art in the table (III) on EDF-Sleep 2013 and EDF-Sleep 2018 database for both Fpz-Cz and Pz-Oz EEG channels. It can be seen that our model outperformed all the existing state-of-art models on $\mathrm{Fpz}-\mathrm{Cz}$ and $\mathrm{Pz}-\mathrm{Oz}$ EEG channels of both databases. The model accuracy is better for Fpz-Cz EEG channel than Pz-Oz EEG channel. It is because the Fpz-Cz EEG channel signals contains more frequency component like delta activity, K-complexes, lower frequency sleep spindles that are useful for sleep staging but Pz-Oz EEG channel signals contains Theta activity and higher frequency sleep spindles [41]. Our proposed model, outperforms existing model(table III) it is because, we have utilized well pre-trained feature learner using triplet loss that is trained to produce embedding of EEG signals with same sleep stage are more closer than embedding of EEG signals with different sleep stage in the Euclidean space.We have utilized Bi-LSTM in both encoder and decoder to capture the sequential dependencies in the signal embedding and self-attention mechanism to focus on most relevant part of the hidden states of the decoder, these techniques are highly beneficial for the better performance of the model.

3) Ablation Studies of Our Proposed Model: We have performed ablation studies on the proposed model by removing some important block to see the contribution of that block in the performance of model. Here, we have examined the contribution of triplet loss based pre-training feature learner $\left(f_{\theta}\right)$ and oversampling of minority classes using SMOTE, one-by-one. The results of ablation studies are summarized and compared with complete proposed model in the table IV, where the mark $(X)$ and $(\checkmark)$ used to represent the removal and presence of particular block in the model, respectively. From the table IV we can see that due to removal of triplet loss based pre-training of $f_{\theta}$, the accuracy of model drops from $94.1071 \%$ to $89.0 \%$ and from $94.0 \%$ to $82.0 \%$ on Fpz-Cz channel of EDF-Sleep-2013 and EDFSleep-2018 respectively and similar kind of drops are observed for another channel of both datasets. So, the triplet loss based pre-training of feature learner $\left(f_{\theta}\right)$ is helping model in learning feature from raw EEG signal for the better the performance. In the another setting of the model, we have removed SMOTE from the both triplet loss based pre-training of $f_{\theta}$ block and classifier to know the contribution of SMOTE in classification on imbalance datasets. We have observe that without oversampling of minority using SMOTE the accuracy drops from $94.1071 \%$ to $91.0 \%$ and $91.0 \%$ to $86.0 \%$ on the Fpz$\mathrm{Cz}$ channel of EDF-Sleep-2013 and EDF-Sleep-2018 respectively and similar kind of drops are observed for another channel of both 


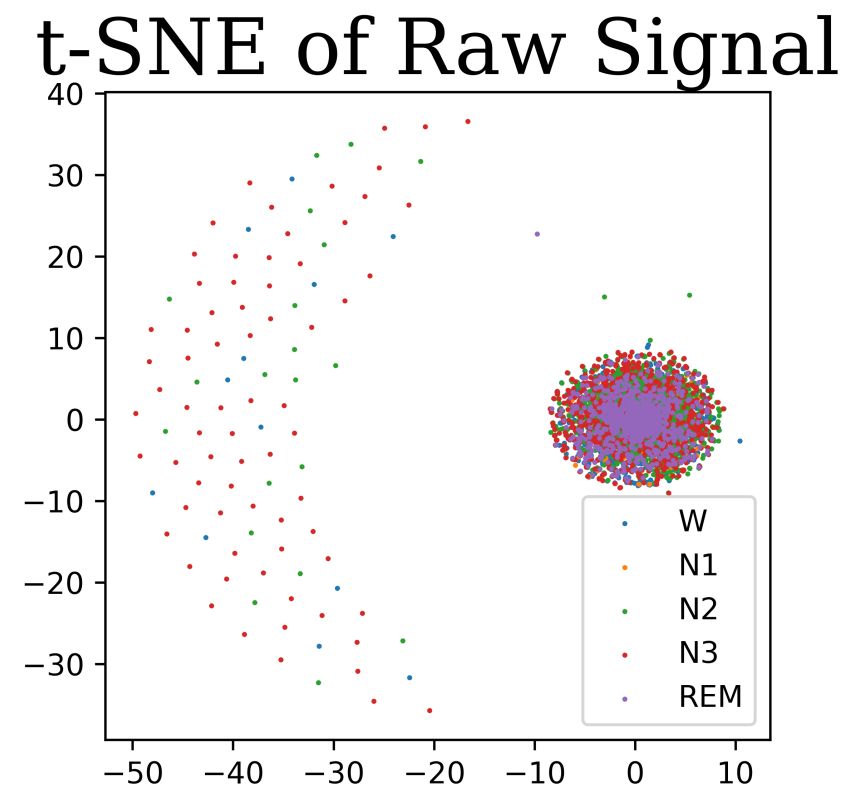

(a)

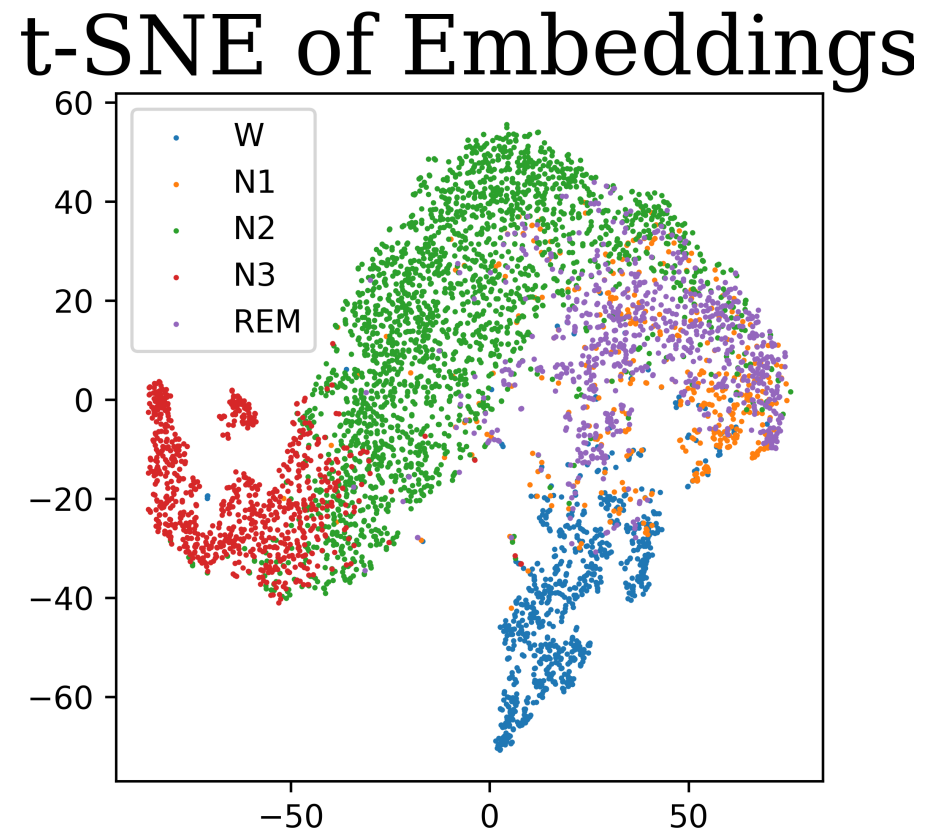

(b)

Fig. 3: Visualization of raw EEG signals and its embedding using t-SNE plot: (a) The t-SNE plot of raw signal(b) The t-SNE plot of embedding of raw signals

TABLE III: Performance comparison of our model with existing state-of-art models

\begin{tabular}{|c|c|c|c|c|c|c|c|c|c|c|c|}
\hline \multirow{2}{*}{ Method } & \multirow{2}{*}{ Dataset } & \multirow{2}{*}{$\mathrm{CV}$} & \multirow{2}{*}{ EEG Channel } & \multicolumn{3}{|c|}{ Overall Performance } & \multicolumn{5}{|c|}{ Per-class Performance (F1) } \\
\hline & & & & $\mathrm{ACC}$ & MF1 & $\kappa$ & $\mathrm{W}$ & N1 & N2 & N3 & REM \\
\hline SCL-SSC & EDF-Sleep-2013 & 20-fold CV & Fpz-Cz & 94.1071 & 92.6416 & 0.9197 & 97.5162 & 84.5873 & 94.7465 & 91.7816 & 94.5765 \\
\hline SleepEEGNet [29] & EDF-Sleep-2013 & 20-fold CV & Fpz-Cz & 84.26 & 79.66 & 0.79 & 89.19 & 52.19 & 86.77 & 85.13 & 85.02 \\
\hline Supratak et. al. [22] & EDF-Sleep-2013 & 20-fold CV & Fpz-Cz & 82.0 & 76.9 & 0.76 & 84.7 & 46.6 & 85.9 & 84.8 & 82.2 \\
\hline Tsinalis et. al. [20] & EDF-Sleep-2013 & 20-fold CV & $\mathrm{Fpz}-\mathrm{Cz}$ & 78.9 & 73.7 & - & 71.6 & 47.0 & 84.6 & 84.0 & 81.4 \\
\hline Tsinalis et. al. [41] & EDF-Sleep-2013 & 20-fold CV & $\mathrm{Fpz}-\mathrm{Cz}$ & 74.8 & 69.8 & - & 65.4 & 43.7 & 80.6 & 84.9 & 74.5 \\
\hline SCL-SSC & EDF-Sleep-2013 & 20-fold CV & $\mathrm{Pz}-\mathrm{Oz}$ & 94 & 92 & 0.9164 & 97 & 84 & 95 & 91 & 95 \\
\hline SleepEEGNet [29] & EDF-Sleep-2013 & 20-fold CV & $\mathrm{Pz}-\mathrm{Oz}$ & 82.83 & 77.02 & 0.77 & 90.27 & 44.64 & 85.74 & 81.55 & 82.88 \\
\hline Supratak et. al. [22] & EDF-Sleep-2013 & 20-fold CV & $\mathrm{Pz}-\mathrm{Cz}$ & 79.8 & 73.1 & 0.72 & 88.1 & 37 & 82.7 & 77.3 & 80.3 \\
\hline SCL-SSC & EDF-Sleep-2018 & 10-fold CV & $\mathrm{Fpz}-\mathrm{Cz}$ & 91.0 & 88.0 & 0.8715 & 95.0 & 74.0 & 92.0 & 89.0 & 91.0 \\
\hline SCL-SSC & EDF-Sleep-2018 & 10-fold CV & $\mathrm{Pz}-\mathrm{Oz}$ & 89.0 & 86.0 & 0.8559 & 95.0 & 70.0 & 91.0 & 87.0 & 90.0 \\
\hline SleepEEGNet [29] & EDF-Sleep-2018 & 10-fold CV & $\mathrm{Fpz}-\mathrm{Cz}$ & 80.03 & 73.55 & 0.73 & 91.72 & 44.05 & 82.49 & 73.45 & 76.06 \\
\hline SleepEEGNet [29] & EDF-Sleep-2018 & 10-fold CV & $\mathrm{Pz}-\mathrm{Oz}$ & 77.56 & 70.00 & 0.68 & 90.26 & 42.21 & 79.71 & 94.83 & 72.19 \\
\hline
\end{tabular}

TABLE IV: Ablation studies of our proposed model

\begin{tabular}{|c|c|c|c|c|c|c|c|c|c|c|c|c|}
\hline \multirow{2}{*}{$\begin{array}{l}\text { Triplet loss based } \\
\text { pre-training of } f_{\theta}\end{array}$} & \multirow{2}{*}{ SMOTE } & \multirow{2}{*}{ Dataset } & \multirow{2}{*}{$\mathrm{CV}$} & \multirow{2}{*}{ EEG Channel } & \multicolumn{3}{|c|}{ Overall Performance } & \multicolumn{5}{|c|}{ Per-class Performance (F1) } \\
\hline & & & & & ACC & MF1 & $\kappa$ & W & N1 & N2 & N3 & REM \\
\hline$\checkmark$ & $\checkmark$ & EDF-Sleep-2013 & 20-fold CV & Fpz-Cz & 94.1071 & 92.6416 & 0.9197 & 97.5162 & 84.5873 & 94.7465 & 91.7816 & 94.5765 \\
\hline$x$ & $\sqrt{\checkmark}$ & EDF-Sleep-2013 & 20-fold CV & Fpz-Cz & 89.0 & 86.0 & 0.8464 & 94 & 68.0 & 91.0 & 87.0 & 88.0 \\
\hline$\checkmark$ & $\checkmark$ & EDF-Sleep-2013 & 20-fold CV & $\mathrm{Pz}-\mathrm{Oz}$ & 94 & 92 & 0.9164 & 97 & 84 & 95 & 91 & 95 \\
\hline$x$ & $\checkmark$ & EDF-Sleep-2013 & 20-fold CV & $\mathrm{Pz}-\mathrm{Oz}$ & 90.0 & 87.0 & 0.8593 & 94.0 & 70.0 & 91.0 & 86.0 & 92.0 \\
\hline$\checkmark$ & $\checkmark$ & EDF-Sleep-2018 & 10 -fold CV & Fpz-Cz & 91.0 & 88.0 & 0.8715 & 95.0 & 74.0 & 92.0 & 89.0 & 91.0 \\
\hline$x$ & $\checkmark$ & EDF-Sleep-2018 & 10-fold CV & Fpz-Cz & 82.0 & 78.0 & 0.7599 & 89.0 & 54.0 & 86.0 & 81.0 & 82.0 \\
\hline$\checkmark$ & $\checkmark$ & EDF-Sleep-2018 & 10-fold CV & $\mathrm{Pz}-\mathrm{Oz}$ & 89.0 & 86.0 & 0.8559 & 95.0 & 70.0 & 91.0 & 87.0 & 90.0 \\
\hline$x$ & $\checkmark$ & EDF-Sleep-2018 & 10-fold CV & $\mathrm{Pz}-\mathrm{Oz}$ & 85.0 & 81.0 & 0.8029 & 91.0 & 57.0 & 88.0 & 83.0 & 87.0 \\
\hline$\checkmark$ & $x$ & EDF-Sleep-2013 & 20-fold CV & Fpz-Cz & 91.0 & 88.0 & 0.8797 & 95.0 & 72.0 & 93.0 & 89.0 & 92.0 \\
\hline$\checkmark$ & $x$ & EDF-Sleep-2013 & 20-fold CV & $\mathrm{Pz}-\mathrm{Oz}$ & 91.0 & 87.0 & 0.8699 & 95.0 & 72.0 & 93.0 & 89.0 & 92.0 \\
\hline$\checkmark$ & $x$ & EDF-Sleep-2018 & 10-fold CV & Fpz-Cz & 86.0 & 82.0 & 0.8120 & 92.0 & 57.0 & 89.0 & 85.0 & 86.0 \\
\hline$\checkmark$ & $x$ & EDF-Sleep-2018 & 10-fold CV & $\mathrm{Pz}-\mathrm{Oz}$ & - & - & - & - & - & - & - & - \\
\hline
\end{tabular}

datasets as summarized in the table IV. Also, we can see that the $F_{1}$ score of the minority class N1 drops from $84.5873 \%$ to $72.0 \%$ and from $74.0 \%$ to $57.0 \%$ on the Fpz-Cz channel of EDF-Sleep2013 and EDF-Sleep-2018 respectively and similar effect observed on another channel of both datasets. So, the oversampling of minority class using SMOTE helping model in reducing the effect of majority and minority class in the datasets.

Although the our model performance is better than existing stateof-art models but there are some limitations with this model. The first limitation is similar to other deep learning model, this model also required large dataset to train the model. The second limitation is it takes longer time in training the model because it processing more 


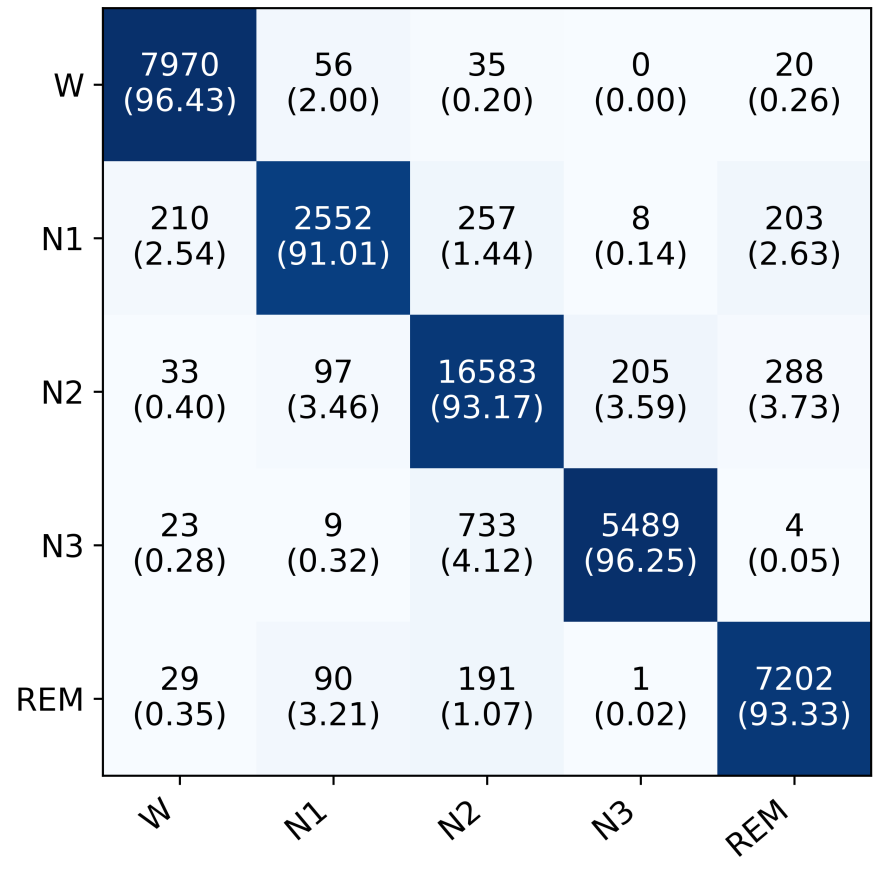

(a)

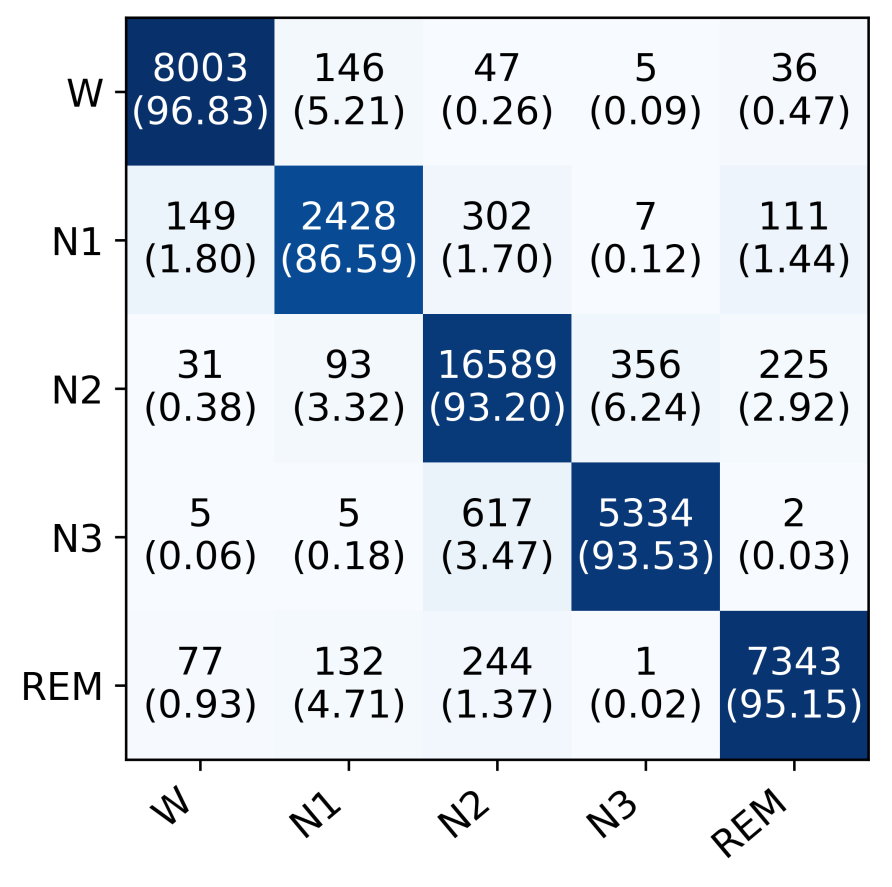

(b)

Fig. 4: Confusion matrix produced from the predicted class by the proposed method on the EDF-Sleep-2013 database using EEG signal from (a)FPz-cz EEG channel (b) Pz-Oz EEG channel

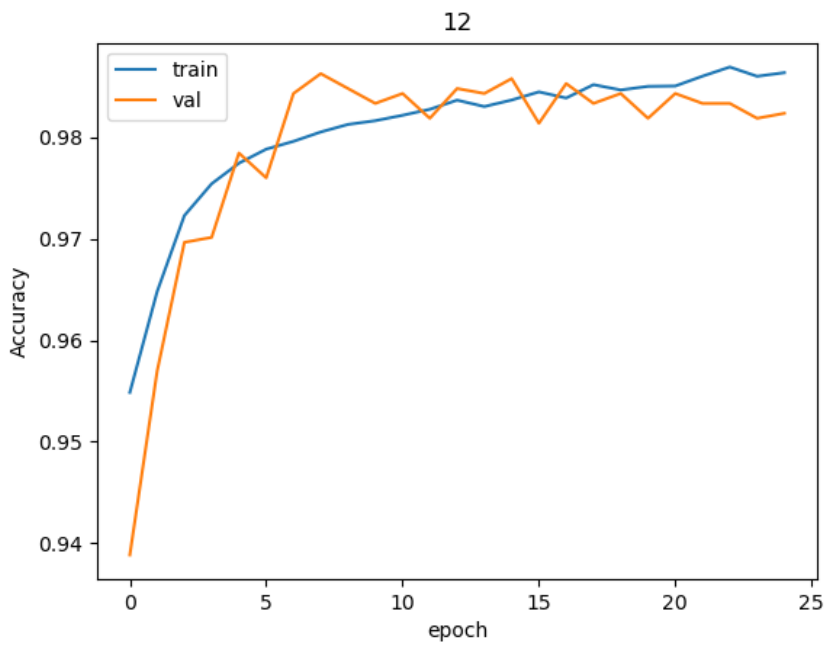

(a)

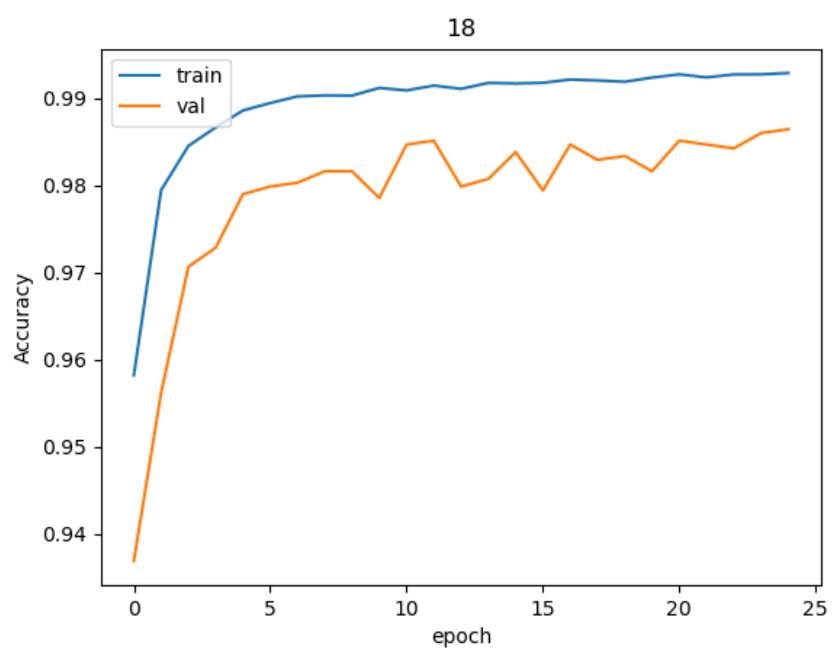

(b)

Fig. 5: Performance graph of proposed model on EDF-Sleep 2013 database in: (a) 12th fold (b) 18th fold

than one epochs in parallel to learn the dependencies in the sequential data. The third limitation is this model trained and evaluated on EEG signals from two available EEG channels only, to evaluate on EEG signals from different EEG channels model need to trained on new EEG signal epochs of that EEG channels.

Furthermore, in future we will extend this work for solving above mentioned limitations of these model and also we will dsign a model that incorporate multimodal polysomnography (PSG) signals including EEG, EOG (electrooculography) and EMG (electromyogram) for better generalization and performance for sleep stage classification.

\section{CONCLUSION}

In this study, we have proposed a novel deep learning model for automatic sleep stage classification. Our model composed of deep network of 1D convolutional neural network as a feature learner to extract features, bidirectional LSTM for building encoder-decoder followed by self-attention mechanism to classify sleep stages.The whole model is trained in two stages: in the first stage, we have trained the feature learner to extract the relevant features from the raw EEG signals using triplet loss and in the second stage, we have 
fine tuned feature learner along with classifier for final classification. We have utilized encoder-decoder based classifier with self-attention mechanism at the output of decoder side. In addition, we have addressed the problem of imbalance dataset by producing synthetic data point for minority classes using SMOTE for oversampling the dataset and also utilized weighted cross-entropy loss function to reduce the affect of imbalance dataset, especially, to boost the model performance for minority sleep stage like N1. The performance comparison table (III) attests that our proposed model outperformed all existing models for automatic sleep stage classification with ceiling level accuracy. The proposed model will not only help the neuro-physiologist for accurate and fast diagnsosis but also will be useful for other biomedical applications to deal with sequential data having imbalance classes.

\section{REFERENCES}

[1] A. Rechtschaffen, "A manual of standardized terminology," Techniques and scoring system for sleep stages of human subjects, 1978.

[2] V. Susić, "Normal sleep," Glas. Srpska akademija nauka i umetnosti. Odeljenje medicinskih nauka, no. 49, pp. 1-6, 2007.

[3] B. M. Altevogt, H. R. Colten et al., "Sleep disorders and sleep deprivation: an unmet public health problem," 2006.

[4] A. C. Krieger, Social and Economic Dimensions of Sleep Disorders, An Issue of Sleep Medicine Clinics, E-Book. Elsevier Health Sciences, 2017, vol. 12, no. 1.

[5] S. Chokroverty et al., "Sleep deprivation and sleepiness," Sleep Disorders Medicine (Third Edition), pp. 22-28, 2009.

[6] C. Iber, "The aasm manual for the scoring of sleep and associated events: Rules," Terminology and Technical Specification, 2007.

[7] R. B. Berry, R. Brooks, C. E. Gamaldo, S. M. Harding, C. Marcus, B. V. Vaughn et al., "The aasm manual for the scoring of sleep and associated events," Rules, Terminology and Technical Specifications, Darien, Illinois, American Academy of Sleep Medicine, vol. 176, p. 2012, 2012.

[8] J. Allan Hobson, "A manual of standardized terminology, techniques and scoring system for sleep stages of human subjects: A. rechtschaffen and a. kales (editors). (public health service, u.s. government printing office, washington, d.c., 1968, 58 p.,4.00)," Electroencephalography and Clinical Neurophysiology, vol. 26, no. 6, p. 644, 1969. [Online]. Available: https://www.sciencedirect.com/science/article/pii/ 0013469469900212

[9] A. Rechtschaffen, "A manual for standardized terminology, techniques and scoring system for sleep stages in human subjects," Brain information service, 1968.

[10] R. Zafar, S. C. Dass, and A. S. Malik, "Electroencephalogram-based decoding cognitive states using convolutional neural network and likelihood ratio based score fusion," PloS one, vol. 12, no. 5, p. e0178410, 2017.

[11] M. Zaeri-Amirani, F. Afghah, and S. Mousavi, "A feature selection method based on shapley value to false alarm reduction in icus a geneticalgorithm approach," in 2018 40th Annual International Conference of the IEEE Engineering in Medicine and Biology Society (EMBC). IEEE, 2018, pp. 319-323.

[12] F. Afghah, A. Razi, R. Soroushmehr, H. Ghanbari, and K. Najarian, "Game theoretic approach for systematic feature selection; application in false alarm detection in intensive care units," Entropy, vol. 20, no. 3, p. 190, 2018.

[13] B. Koley and D. Dey, "An ensemble system for automatic sleep stage classification using single channel eeg signal," Computers in biology and medicine, vol. 42, no. 12, pp. 1186-1195, 2012.

[14] L. Fraiwan, K. Lweesy, N. Khasawneh, H. Wenz, and H. Dickhaus, "Automated sleep stage identification system based on time-frequency analysis of a single eeg channel and random forest classifier," Computer methods and programs in biomedicine, vol. 108, no. 1, pp. 10-19, 2012.

[15] Y.-L. Hsu, Y.-T. Yang, J.-S. Wang, and C.-Y. Hsu, "Automatic sleep stage recurrent neural classifier using energy features of eeg signals," Neurocomputing, vol. 104, pp. 105-114, 2013.

[16] K. Simonyan and A. Zisserman, "Very deep convolutional networks for large-scale image recognition," arXiv preprint arXiv:1409.1556, 2014.

[17] J. Yue-Hei Ng, M. Hausknecht, S. Vijayanarasimhan, O. Vinyals, R. Monga, and G. Toderici, "Beyond short snippets: Deep networks for video classification," in Proceedings of the IEEE conference on computer vision and pattern recognition, 2015, pp. 4694-4702.
[18] D. Bahdanau, K. Cho, and Y. Bengio, "Neural machine translation by jointly learning to align and translate," arXiv preprint arXiv:1409.0473, 2014.

[19] Y. Kim, "Convolutional neural networks for sentence classification," in Proceedings of the 2014 Conference on Empirical Methods in Natural Language Processing (EMNLP). Doha, Qatar: Association for Computational Linguistics, Oct. 2014, pp. 1746-1751. [Online]. Available: https://aclanthology.org/D14-1181

[20] O. Tsinalis, P. M. Matthews, and Y. Guo, "Automatic sleep stage scoring using time-frequency analysis and stacked sparse autoencoders," Annals of biomedical engineering, vol. 44, no. 5, pp. 1587-1597, 2016.

[21] S. Chambon, M. N. Galtier, P. J. Arnal, G. Wainrib, and A. Gramfort, "A deep learning architecture for temporal sleep stage classification using multivariate and multimodal time series," IEEE Transactions on Neural Systems and Rehabilitation Engineering, vol. 26, no. 4, pp. 758-769, 2018.

[22] A. Supratak, H. Dong, C. Wu, and Y. Guo, "Deepsleepnet: A model for automatic sleep stage scoring based on raw single-channel eeg," IEEE Transactions on Neural Systems and Rehabilitation Engineering, vol. 25, no. 11, pp. 1998-2008, 2017.

[23] E. P. Giri, M. I. Fanany, and A. M. Arymurthy, "Combining generative and discriminative neural networks for sleep stages classification," arXiv preprint arXiv:1610.01741, 2016.

[24] A. Sathyanarayana, S. Joty, L. Fernandez-Luque, F. Ofli, J. Srivastava, A. Elmagarmid, T. Arora, and S. Taheri, "Sleep quality prediction from wearable data using deep learning," JMIR mHealth and uHealth, vol. 4, no. 4, p. e125, 2016.

[25] Y. Wang and D. Wu, "Deep learning for sleep stage classification," in 2018 Chinese Automation Congress (CAC). IEEE, 2018, pp. 38333838.

[26] H. Dong, A. Supratak, W. Pan, C. Wu, P. M. Matthews, and Y. Guo, "Mixed neural network approach for temporal sleep stage classification," IEEE Transactions on Neural Systems and Rehabilitation Engineering, vol. 26, no. 2, pp. 324-333, 2017.

[27] A. Sano, W. Chen, D. Lopez-Martinez, S. Taylor, and R. W. Picard, "Multimodal ambulatory sleep detection using 1 stm recurrent neural networks," IEEE journal of biomedical and health informatics, vol. 23, no. 4, pp. 1607-1617, 2018.

[28] H. Phan, F. Andreotti, N. Cooray, O. Y. Chén, and M. De Vos, "Seqsleepnet: end-to-end hierarchical recurrent neural network for sequence-tosequence automatic sleep staging," IEEE Transactions on Neural Systems and Rehabilitation Engineering, vol. 27, no. 3, pp. 400-410, 2019.

[29] S. Mousavi, F. Afghah, and U. R. Acharya, "Sleepeegnet: Automated sleep stage scoring with sequence to sequence deep learning approach," PloS one, vol. 14, no. 5, p. e0216456, 2019.

[30] A. I. Humayun, A. S. Sushmit, T. Hasan, and M. I. H. Bhuiyan, "Endto-end sleep staging with raw single channel eeg using deep residual convnets," in 2019 IEEE EMBS International Conference on Biomedical \& Health Informatics (BHI). IEEE, 2019, pp. 1-5.

[31] E. Eldele, Z. Chen, C. Liu, M. Wu, C.-K. Kwoh, X. Li, and C. Guan, "An attention-based deep learning approach for sleep stage classification with single-channel eeg," IEEE Transactions on Neural Systems and Rehabilitation Engineering, vol. 29, pp. 809-818, 2021.

[32] M. X. Cohen, Analyzing neural time series data: theory and practice. MIT press, 2014

[33] M. Schultz and T. Joachims, "Learning a distance metric from relative comparisons," Advances in neural information processing systems, vol. 16, pp. 41-48, 2004.

[34] F. Schroff, D. Kalenichenko, and J. Philbin, "Facenet: A unified embedding for face recognition and clustering," in Proceedings of the IEEE Conference on Computer Vision and Pattern Recognition (CVPR), June 2015.

[35] M.-T. Luong, H. Pham, and C. D. Manning, "Effective approaches to attention-based neural machine translation," arXiv preprint arXiv:1508.04025, 2015.

[36] S. Wang, W. Liu, J. Wu, L. Cao, Q. Meng, and P. J. Kennedy, "Training deep neural networks on imbalanced data sets," in 2016 international joint conference on neural networks (IJCNN). IEEE, 2016, pp. 43684374.

[37] B. Kemp, A. H. Zwinderman, B. Tuk, H. A. Kamphuisen, and J. J. Oberye, "Analysis of a sleep-dependent neuronal feedback loop: the slow-wave microcontinuity of the eeg," IEEE Transactions on Biomedical Engineering, vol. 47, no. 9, pp. 1185-1194, 2000.

[38] A. L. Goldberger, L. A. Amaral, L. Glass, J. M. Hausdorff, P. C. Ivanov, R. G. Mark, J. E. Mietus, G. B. Moody, C.-K. Peng, and H. E. Stanley, "Physiobank, physiotoolkit, and physionet: components of a new 
research resource for complex physiologic signals," circulation, vol. 101, no. 23, pp. e215-e220, 2000.

[39] W. Bowyer Kevin, O. Hall Lawrence, and W. Philip Kegelmeyer, "Synthetic minority over-sampling technique," J Artif Intell Res, vol. 16, pp. 321-57, 2002.

[40] L. Van der Maaten and G. Hinton, "Visualizing data using t-sne.” Journal of machine learning research, vol. 9, no. 11, 2008.

[41] O. Tsinalis, P. M. Matthews, Y. Guo, and S. Zafeiriou, "Automatic sleep stage scoring with single-channel eeg using convolutional neural networks," arXiv preprint arXiv:1610.01683, 2016. 